ebenen, die in digitalen Gesellschaften in dieser Form kaum standhaft sind. Vielmehr scheinen die Ebenen der Spontan-, Versammlungs- und Medienöffentlichkeit vor allem in der digitalen Sphäre zunehmend miteinander zu verschmelzen. Zudem stellt sich die Frage, welche Akteur_innen die Prozessschritte hin zur Informationsveröffentlichung durchführen und ob die Kybernetik des Systems durch alternative Akteur_innen, die sich eine Verbreitung von Informationen selbst auferlegt, standhält (vgl. Kapitel II.3).

Gesamthaft betrachtet scheinen alle drei Theorieperspektiven wesentlich für die Beschreibung öffentlicher Kommunikation in modernen, digitalen Gesellschaften. Eine normative Perspektive zeigt das Soll - also wie öffentliche Kommunikation funktionieren sollte. Die systemtheoretische Perspektive gibt Aufschluss über die Funktionen von Öffentlichkeit und den Massenmedien. Der Gedanke einer binären Codierung ist insofern wesentlich, als auch Online-Kommunikation in Form von Codes oder Logiken operiert - im Sinne von: Was hat Nachrichtenwert? Wie steuern Plattformen öffentliche Kommunikation (hierzu ausführlicher in Kapitel II.3)? Eine analytische Betrachtung repräsentiert eine dazwischenliegende Betrachtung, die sowohl normative als auch systemtheoretische Indizien enthält. Fraglich ist in diesem Zusammenhang vor allem die Unterscheidung in Öffentlichkeitsebenen, die sich in digitalen Sphären zunehmend überschneiden.

Die Einordnung der unterschiedlichen Theorieperspektiven ist vor allem deshalb wesentlich, da von einem Wandel der öffentlichen Kommunikation durch die Digitalisierung auszugehen ist. Alle beschriebenen Theorien beziehen sich auf die Zeit vor dem 21. Jahrhundert, das - aus einer Medienperspektive - vor allem durch die Digitalisierung geprägt ist. Das folgende Kapitel skizziert den Wandel der Öffentlichkeit, bevor aktuelle öffentlichkeitstheoretische Aspekte und eine Neu-Einordnung bisheriger Theoriefiguren erfolgt. $\mathrm{Zu}$ diesem Zweck wird auf Habermas' (2018 [1962]) Ausführungen zum Strukturwandel der Öffentlichkeit eingegangen. Habermas' Theorie skizziert die Historie der öffentlichen Kommunikation, was in Anbetracht der Frage, wie digitale Öffentlichkeiten zu skizzieren sind und ob es Neudefinitionen hinsichtlich des Öffentlichkeitsbegriffes bedarf, wesentlich ist.

\title{
II.2 Der Strukturwandel der Öffentlichkeit
}

Habermas (2018 [1962]) beschreibt in seinem Werk «Strukturwandel der Öffentlichkeit» wesentliche historische Eckpfeiler, wie Öffentlichkeit in den vergangenen Jahrhunderten hergestellt wurde und sich schliesslich veränderte. Habermas' Theorie ist nicht zuletzt ob seiner definitorischen Stärken hinsichtlich des Öffentlichkeitsbegriffes für die kommunikationswissenschaftliche und soziologische 
Öffentlichkeitsforschung von hoher Relevanz, sondern vor allem wegen seiner historischen Aufarbeitung, die zu einer Reflexion von Öffentlichkeit in digitalen Gesellschaften einlädt.

Jürgen Habermas skizziert - wie bereits im vorigen Kapitel erörtert - einen Idealtypus von Öffentlichkeit und nimmt dabei Bezug auf die bürgerliche Öffentlichkeit des 17. und 18. Jahrhunderts. Die «bürgerliche Öffentlichkeit» bezieht sich auf Versammlungen von Privatleuten, ursprünglich im Bereich der Familie und später in Kaffeehäusern und Salons, die als Zentren literarischer und politischer Öffentlichkeit gesehen werden können. Leitend war dabei der Gedanke des öffentlichen Räsonnements, also die Berufung auf die Vernunft (Habermas, 2018 [1962], S. 86). Die politische Öffentlichkeit vermittelt zwischen der Sphäre des Privaten, im Sinne der bürgerlichen Gesellschaft, und der Sphäre der öffentlichen Gewalt, also dem Staat. Habermas streicht durch seine Ausführungen die vor allem in der Soziologie prominent thematisierte Unterscheidung zwischen Öffentlichkeit und Privatsphäre heraus, wobei sich letztere auf die bürgerliche Gesellschaft im engeren Sinn bezieht, wie die familiäre Intimsphäre, den Bereich des Warenverkehrs oder der gesellschaftlichen Arbeit (Habermas, 2018 [1962], S. 89f.). Im 18. Jahrhundert entwickelte sich die öffentliche Meinung durch Institutionen der Kunst-, Literatur-, Theater- und Musikkritik weiter, bis schliesslich die Zeitschrift zum publizistischen Kritikinstrument wurde, zunächst in Form kunstund kulturkritischer Journale. Die Presse und ihre Kritik lösten entsprechend die Kaffeehäuser, Salons und Tischgesellschaften als Vermittlungsinstanzen des Publikums ab. In dieser Zeit entwickelte sich innerhalb der bürgerlichen Öffentlichkeit auch zunehmend ein politisches Bewusstsein, wonach die öffentliche Meinung «als die einzig legitime Quelle» (Habermas, 2018 [1962], S. 119) der Gesetze galt. Das öffentliche Räsonnement, die Vernunft, bildete sich dabei unabhängig von sozialen und politischen Rängen, basierend auf «der Kraft des besseren Arguments» (Habermas, 2018 [1962], S. 119). Im 18. Jahrhundert entwickelte sich, zunächst in Grossbritannien und später in Frankreich, zudem nach und nach der selbständige Journalismus, als Dirigent der öffentlichen Meinung. Journalismus war fortan davon geprägt, eine Kommentierung, kritische Position und öffentliche Opposition gegenüber der Regierung einzunehmen, und galt gleichsam als kritisches Organ des räsonierenden Publikums, im Sinne eines «fourth Estate» (Habermas, 2018 [1962], S. 126).

Dieses Idealbild der Öffentlichkeit ändert sich Habermas zufolge (2018 [1962]) im Zuge des sozialen Struktur- und politischen Funktionswandels. Habermas erachtet die Trennung von privater und öffentlicher Sphäre als wesentliche Grundvoraussetzung für eine funktionierende Öffentlichkeit. Diese wurde im Zuge des sozialen Strukturwandels ab dem späteren 19. Jahrhundert brüchig, da es zu Eingriffen des Staates respektive der öffentlichen Gewalt in die gesellschaftliche Sphäre kam. Sozialstaatliche Massnahmen führten zu einer Verschränkung 
der privaten und öffentlichen Sphäre. Weiter sieht Habermas (2018 [1962], S. 257) einen «Zerfall einer literarischen Öffentlichkeit» durch «die Ablösung eines kulturell räsonierenden Lesepublikums durch das Massenpublikum der Kulturkonsumenten». Entsprechend entwickelte sich auch die Presse vom politischen Format zur kommerziellen Massenpresse (oder Sensationspresse) - nicht zuletzt, um breiteren Bevölkerungsschichten einen Zugang zur Öffentlichkeit zu gewähren. Habermas (2018 [1962], S. 259) schätzt den sozialen Strukturwandel wie folgt ein:

Der Kulturkonsum ist freilich von literarischer Vermittlung in hohem Maße entlastet; nicht-verbale Mitteilungen oder solche, die, wenn nicht überhaupt in Bild und Ton übersetzt, durch optische und akustische Stützen erleichtert sind, verdrängen in mehr oder minder großem Maße die klassischen Formen der literarischen Produktion.

Dieses Zitat verdeutlicht mit Blick auf die Gegenwart, dass der soziale Strukturwandel nicht nur bis heute seine Spuren hinterlässt, sondern sich dahingehend noch weiter in diese Richtung (z. B. der Audiovisualisierung) entwickelt hat, was als potentielle Gefahr für die demokratische Funktion des Journalismus und dessen Qualität zu werten ist. Auch aus Publikumssicht scheint somit das «Räsonnement [...] tendenziell dem >Geschmacks-< und >Neigungsaustausch $<$ von Konsumenten» auszuweichen (Habermas, 2018 [1962], S. 261), womit sich die Nachfrage des Publikums an Aufmerksamkeitswerte richtet. Insofern spricht Habermas (2018 [1962]) gleichbedeutend von einer Privatisierung des Öffentlichen, wonach vor allem die Berichterstattung über private Lebensgeschichten an Zugkraft und Aufmerksamkeit gewinnt. Auch im privaten Bereich stellt Habermas eine Veränderung fest, insofern, als die literarische Ausdruckform im Generellen wie der Briefwechsel und die Buchlektüre durch den Konsum der Massenmedien (wie Zeitungsredaktionen und Fernsehstationen) an Bedeutung verloren habe. Der Prozess vom «kulturräsonierenden zum kulturkonsumierenden Publikum» (Habermas, 2018 [1962], S. 267) impliziert gleichzeitig eine «neue» Funktion der Öffentlichkeit, nämlich in Form der Integration von Werbung und Public Relations: «Je mehr sie [die Öffentlichkeit] als Medium politischer und ökonomischer Beeinflussung eingesetzt werden kann, um so unpolitischer wird sie im ganzen und dem Schein nach privatisiert.» (Habermas, 2018 [1962], S. 267). Diese zunehmende Privatisierung und Implementierung privater Interessen sind folglich als wesentlicher Rückschritt (oder Desorganisation) des Idealbildes einer demokratischen Öffentlichkeit zu betrachten, da die bürgerliche Öffentlichkeit im Habermasschen Sinne von der Trennung zwischen öffentlichem und privatem Bereich lebt.

Neben dem sozialen Wandel spricht Habermas (2018 [1962]) weiter von einem politischen Funktionswandel. Er argumentiert hier auf Basis der eben beschriebenen Kommerzialisierung des Mediensystems, in dem private und öffentliche 
Interessen zunehmend verschwimmen würden. Der Autor spricht dabei von einem Ende der Öffentlichkeit, wenn öffentliche Institutionen (wie die Medien selbst) ihre Unabhängigkeit verlieren würden und auf den politischen Bereich angewiesen bzw. von diesem abhängig wären. Die Presse, ursprünglich auf dem Räsonnement des Publikums basierend und als Art verlängerter Arm der Bürger_ innen fungierend, entwickele sich im Zuge der Kommerzialisierung zu einer Verlängerung politischer und wirtschaftlicher Interessen. Insofern könne die Presse auch ihre kritische Funktion zunehmend verlieren.

Habermas bezieht sich in seiner Schrift «Strukturwandel der Öffentlichkeit» (2018 [1962]) auf die Entwicklung der Öffentlichkeit in den vergangenen Jahrhunderten bis hin zum 20. Jahrhundert. Dabei handelt es sich um detaillierte theoretische und historische Beobachtungen des Mediensystems, die mit Blick auf aktuelle Entwicklungen im 21. Jahrhundert noch immer in hohem Masse griffig sind. Dennoch brachten der digitale Wandel und die Etablierung neuer Medien erneut die Frage hervor, inwiefern sich die öffentliche Kommunikation weiterentwickelt. In diesem Kontext wird in aktueller, vor allem theoretischer Forschung häufig von einer digitalen Transformation oder einem digitalen Strukturwandel der Öffentlichkeit gesprochen (z. B. Eisenegger, 2017; Fraser, 2010; Hagen, Wieland \& In der Au, 2017; Helmond, 2015; Thiel, 2016). Diese Annahmen basieren in erster Linie auf der Annahme einer Veränderung des öffentlichen Raumes aufgrund neuer Möglichkeiten der öffentlichen Kommunikation über das Internet oder über Social-Media-Plattformen. Für die vorliegende Arbeit ist diesbezüglich von einer zentralen theoretischen Überlegung zu sprechen, die eine Neudeutung des Öffentlichkeitsund Gegenöffentlichkeitsbegriffs in Frage stellt. Das folgende Kapitel gibt daher einen Überblick über aktuelle theoretische Überlegungen zum Öffentlichkeitsbegriff, wobei vor allem auf die Veränderung des Mediensystems respektive der Öffentlichkeit basierend auf dem digitalen Wandel eingegangen wird.

\section{II.3 Die digitale Transformation der Öffentlichkeit}

Habermas' Ausführungen zum Strukturwandel der Öffentlichkeit laden dazu ein, diese für das 21. Jahrhundert und allenfalls zukünftige Entwicklungen weiterzudenken. In aktuellen theoretischen Arbeiten wird in diesem Zusammenhang oftmals von einem dritten Strukturwandel der Öffentlichkeit oder einer digitalen Transformation der Öffentlichkeit gesprochen (z. B. Eisenegger, 2017; Fraser, 2010; Hagen et al., 2017; Helmond, 2015; Thiel, 2016). Der erste und der zweite Strukturwandel lassen sich von Habermas (2018 [1962]), Münch (1995) oder Imhof (2000) ableiten (vgl. hierzu auch Eisenegger, 2021). Zusammenfassend lässt sich der erste Strukturwandel der Öffentlichkeit im 18. und 19. Jahrhundert verorten, als durch das Leitmedium der Zeitung erstmalig Öffentlichkeit massenmedial hergestellt 